\title{
Network pharmacology study of vaccarin for the prevention and treatment of postmenopausal osteoporosis
}

\section{Mengying Bao}

Guangxi Medical University https://orcid.org/0000-0003-0258-1059

\section{Yan Dai}

The Second Afficiated Hospital of Guangxi Medical University

\section{Xiaojun Chen}

Guangzhou University of Chinese Medicine

\section{Shijie Liao}

Guangxi Medical University First Affiliated Hospital

\section{Wenyu Feng}

Guangxi Medical University First Affiliated Hospital

\section{Chengsen Lin}

Guangxi Medical University First Affiliated Hospital

\section{Yu Ye}

The Second Affiliated Hospital of Guangxi Medical University

\section{Zengnan Mo}

Guangxi Medical University

Yun Liu ( $\square$ liuyun200450250@sina.com )

Guangxi Medical University First Affiliated Hospital https://orcid.org/0000-0002-7745-1083

\section{Research}

Keywords: Vaccarin, Osteoporosis, Network pharmacology, Mechanism of action

Posted Date: September 22nd, 2020

DOl: https://doi.org/10.21203/rs.3.rs-79318/v1

License: (c) (i) This work is licensed under a Creative Commons Attribution 4.0 International License. Read Full License 


\section{Abstract}

Background: As the main active ingredient of Semen Vaccariae, vaccarin is a flavonoid glycoside useful for the prevention and treatment of numerous diseases. Our previous study found that vaccarin can reduce osteolysis-induced titanium by inhibiting osteoclast formation. However, the issue of whether vaccarin can prevent and treat postmenopausal osteoporosis remains unclear.

Method: In this study, we explored the mechanism of action of vaccarin for the prevention of postmenopausal osteoporosis via a network pharmacological approach. We identified the intersecting targets of osteoporosis-related genes retrieved from multiple disease target databases, as well as targets of potential action of vaccarin retrieved from drug-related databases. We then used the intersectional targets to establish a protein-protein interaction (PPI) network. Finally, we performed bioinformatics analysis to enrich Gene Ontology (GO) biological processes and Kyoto Encyclopedia of Genes and Genomes (KEGG) pathways.

Results $\llbracket A$ total of 28 cross targets of vaccarin and osteoporosis were identified. PPI network analysis identified six target proteins, namely, IL-6, TNF, VEGFA, HSP90AA1, CREB1, and IL-2, which may be the key targets of vaccarin against osteoporosis. The 28 intersectional targets were mainly involved in 23 biological processes, such as regulation of apoptosis, positive regulation of neovascularization, and angiogenesis, whereas KEGG enrichment analysis revealed that they were primarily related to 22 different signaling pathways, such as PI3K/Akt pathway, cancer pathway, hepatitis B pathway, and tuberculosis pathway.

Conclusion: We used a network pharmacology approach to predict the key targets of vaccarin for the prevention of osteoporosis from a systems perspective. We determined that the signaling pathways were chiefly engaged in different pathological processes affecting differentiation and apoptosis of bone rebuilding cells, endocrine metabolic disorders, inflammatory responses, and other disease interactions. This study provides a theoretical basis and therapeutic ideas for the treatment of postmenopausal osteoporosis and offers promising directions for further research on the regulatory mechanism of vaccarin.

\section{Introduction}

Semen Vaccariae, the dried ripe seeds of the Vaccaria hispanica (Mill.) Rauschert, is a traditional Chinese herbal medicine that has been used in China for thousands of years[1].. It is bitter with a neutral $\mathrm{pH}$; it is distributed to the liver and stomach; and it has the effect of activating blood circulation, decreasing lower breast swelling, and promoting diuresis and lactogenesis[2]. This herbal medicine is mainly used in the clinical treatment of lactating difficulties in postpartum women, breast carbuncle swelling and pain, menstrual disorder, gonorrhea astringent pain, and other diseases[1, 3]. Modern technology can isolate various types of compounds from the extract of Semen Vaccariae, such as cyclic peptides, triterpenes, saponins, and flavonol glycosides[4]. 
As the main active ingredient of Semen Vaccariae, vaccarin is a flavonoid glycoside and a phytoestrogen[5]. Previous studies on vaccarin largely focused on its extraction and separation, drug metabolism, pharmacological mechanism, cell signaling, and other aspects. Numerous studies have confirmed the effectiveness of vaccarin in the prevention and treatment of many diseases, such as in reducing endothelial cell damage[6, 7], promoting angiogenesis[8], inhibiting inflammation, preventing atherosclerosis[9], promoting wound healing[10, 11], improving insulin resistance[12], and preventing renal hypertension and cardiovascular remodeling[13]. Through extensive basic experiments, Professor Qiu Liying's team found that vaccarin can prevent and treat diseases via different targets and signaling pathways. Another study reported that vaccarin can reverse kidney damage from hypertension by reducing inflammation, oxidative stress, and fibrosis[13].

In our previous study, we found that vaccarin can effectively prevent titanium particle-induced pathological osteolysis in vivo by blocking the MAPK and NF-K $\beta$ signaling pathways[14]. In terms of pathogenesis, the titanium particle-induced osteolysis model, a model that mimics the common clinical condition of periprosthetic aseptic loosening, induced the loss of periprosthetic bone mass through wear particle-mediated aseptic inflammation. Postmenopausal osteoporosis is primarily due to a sharp decline in estrogen, leading to metabolic disorders caused by bone loss [15]. In this manner, postmenopausal osteoporosis is similar to titanium particle-induced osteolysis in terms of pathogenesis. Nevertheless, these two conditions have striking differences. Thus, the issue of whether vaccarin can also prevent and treat postmenopausal osteolysis is not yet clear.

Various methods, such as osteoclast formation experiments and luciferase gene reporting experiments on the NFAT or NF-к $\beta$ signaling pathway, are available for screening compounds or herbal monomers that are effective in the prevention and treatment of osteoporosis. However, a large number of drugs in the early stage must be bought, and constructing luciferase gene cell lines and purchasing reagents are timeconsuming and expensive. Therefore, a simple, rapid, and economical screening method for selecting effective drugs for a particular disease must be developed.

The concept of "network pharmacology" was first proposed by the British scholar Andrew L. Hopkins in 2007[16]. This concept is based on the similarity between drugs and drugs in structure, efficacy, and other aspects to predict the potential target genes of the active pharmaceutical ingredients. By combining the multiple interactions of target molecules and biological effector molecules, the efficacy of drugs and the biological functions and signaling pathways involved in drug treatment can be predicted. Compared with Western medicine, traditional Chinese herbal medicine has the advantages of strong plant origin, easy access, and low toxicity, and thus it enjoys high trustworthiness among the general public. However, it also has shortcomings, such as complex compositions and unknown mechanisms of action, which are the primary reasons that hinder the recognition and further promotion of Chinese herbal medicine worldwide. The research and development model of Chinese herbal medicine is different from that of Western medicine. The herbal active monomer is the main effective part of the herbal compound, and this monomer often acts as a disease-modifying agent by regulating multiple targets and pathways. The emerging field of network pharmacology largely focuses on multitarget and multipathway action 
characteristics of the active monomers of Chinese herbal medicines. Through this approach, the potential mechanism of action of the active monomers at the gene and protein levels can be revealed. This approach has bridged the modernization and internationalization of traditional Chinese medicine and addressed the challenges in research on Chinese herbal medicines.

Research on osteoporosis via network pharmacology is still in its initial stage and mainly focuses on the compounds of Chinese herbal medicines. Rhizoma drymariae, Er-xian decotion, and Sinomenii Caulis are folk Chinese herbs with good clinical effects commonly used for the treatment of osteoporosis and with relevant network pharmacology studies[17-19]. By contrast, the network pharmacology of Semen Vaccariae and its active ingredient vaccarin for the treatment of osteoporosis is lacking. This study conducted bioinformatics analysis via a drug component-target gene-disease pathway network to explore the pharmacological mechanisms of vaccarin in relation to osteoporosis and provide a theoretical basis for further study of vaccarin for osteoporosis prevention and clinical translational applications.

\section{Material And Methods}

\subsection{Study design}

Genes associated with osteoporosis were searched from multiple disease target databases, whereas the potential targets of vaccarin were retrieved from drug-related databases. The cross targets between the genes and the potential targets were then identified. Multiple public databases were consulted to analyze the cross-target networks and enrichment pathways to explore the major signaling pathways of vaccarin and its molecular networks involved in the prevention and treatment of osteoporosis. The workflow is shown in Fig. 1.

\subsection{Prediction of vaccarin targets}

SwissTargetPrediction (http://www.swisstargetprediction.ch/), SEA (http://sea.bkslab.org/search/), and TargetNet (http://targetnet.scbdd.com/) were used to retrieve the potential targets of vaccarin with the "human" species setting, and then duplicate data were eliminated to obtain the final potential targets of vaccarin.

\subsection{Mining of osteoporosis targets}

Comparative Toxicogen-omics Database (http://ctdbase.org/), Genecards (http://genecards.org/), Therapeutic Target Database (http://db.idrblab.net/ttd/), OMIM (https://www.omim.org/), and PharmGKB (https://www.pharmgkb.org/) were searched for information on osteoporosis-related genes and target proteins.

\subsection{Establishing a network of protein-protein interactions (PPI)}

Drug-disease crossover genes were screened and imported into the String database (http://string .db.org/). The species was defined as "human" to construct the vaccarin for osteoporosis protein-protein 
interaction (PPI) network. Finally, network node and edge information were entered into Cytoscape3.6.0 for PPI network visualization.

\subsection{Bioinformatics annotation and pathway analysis of crossover targets}

Metascape database (http://metascape.org/gp/index.html\#/main/step1) [20] and Kyoto Encyclopedia of Genes and Genomes (KEGG) pathways[21] (https://www.genome.jp/kegg/pathway.html) were utilized to retrieve information about the functional annotation of the drug-disease crossover genes and extract the typical pathways highly associated with these proteins. The relevant pathways were then filtered with Pvalue $<0.05$ and mapped using the R3.2.5 software.

\section{Results}

\subsection{Analysis of crossover targets}

The SEA, Swiss, and TargetNet databases were used to retrieve 56, 15, and 20 possible targets for vaccarin, respectively. Only 76 genes remained after exclusion of duplicate data were excluded. The osteoporosis targets were searched in the Comparative Toxicogenomics, Genecards, Therapeutic target OMIM, and PharmGKB databases, leaving a total of 3601 target proteins after eliminating duplicate data. The set of drug targets and disease target proteins and their relationships were represented in a closedloop form at fixed positions to obtain Venn diagrams, as shown in Fig. 2, and 28 intersecting target proteins were found, with specific target genes, target proteins, associated databases and scores shown in Table 1. 
Table 1

Analysis of common targets between vaccarin and osteoporosis

\begin{tabular}{|c|c|c|c|}
\hline ID & Target genes & Target proteins & Database and scores \\
\hline 1 & CREB1 & Cyclic AMP-responsive element-binding protein 1 & $\operatorname{SEA}(111.407)$ \\
\hline 2 & FGF1 & Fibroblast growth factor 1 & SEA(77.350) \\
\hline 3 & FASN & Fatty acid synthase & SEA(77.350) \\
\hline 4 & TYR & Tyrosinase & SEA(65.708) \\
\hline 5 & $\mathrm{P} 4 \mathrm{HB}$ & Protein disulfide-isomerase & SEA(60.050 \\
\hline 6 & NRAS & GTPase Nras & SEA(54.040) \\
\hline 7 & PTPRS & Receptor-type tyrosine-protein phosphatase S & SEA(50.396) \\
\hline 8 & FGF2 & Fibroblast growth factor 2 & SEA(26.341) \\
\hline 9 & CYP1B1 & Cytochrome P450 1B1 & SEA(23.156) \\
\hline 10 & VEGFA & Vascular endothelial growth factor A & SEA(19.070) \\
\hline 11 & LGALS3 & Galectin-3 & SEA(18.630) \\
\hline 12 & ERAP1 & Endoplasmic reticulum aminopeptidase 1 & SEA(14.186) \\
\hline 13 & ALPI & Intestinal-type alkaline phosphatase & SEA(11.618) \\
\hline 14 & IL6 & Interleukin-6 & SEA(10.691) \\
\hline 15 & ABCB1 & Multidrug resistance protein 1 & SEA(10.059) \\
\hline 16 & ADRA2A & Alpha-2a adrenergic receptor & SWISS(0.0499) \\
\hline 17 & CA2 & Carbonic anhydrase II & SWISS(0.0499) \\
\hline 18 & TNF & TNF-alpha & SWISS(0.0499) \\
\hline \multirow[t]{2}{*}{19} & IL2 & Interleukin-2 & SEA(51.286) \\
\hline & & & SWISS(0.0499) \\
\hline \multirow[t]{2}{*}{20} & $\mathrm{XDH}$ & Xanthine dehydrogenase/oxidase & $\mathrm{SEA}(36.443) \square$ \\
\hline & & & SWISS(0.0499) \\
\hline \multirow[t]{2}{*}{21} & $\mathrm{ACHE}$ & Acetylcholinesterase & $\operatorname{SEA}(28.721) \square$ \\
\hline & & & SWISS(0.0499) \\
\hline \multirow[t]{2}{*}{22} & NMUR2 & Neuromedin-U receptor 2 & SEA (14.777)] \\
\hline & & & SWISS(0.0499) \\
\hline 23 & DNMT1 & DNA (cytosine-5)-methyltransferase 1 & TargetNet(0.999) \\
\hline
\end{tabular}




\begin{tabular}{|llll|}
\hline ID & Target genes & Target proteins & Database and scores \\
\hline 24 & PTGS1 & Prostaglandin G/H synthase 1 & TargetNet(0.991) \\
\hline 25 & BCL2A1 & Bcl-2-related protein A1 & TargetNet(0.987) \\
\hline 26 & CYP1A2 & Cytochrome P450 1B1 & TargetNet(0.822) \\
\hline 27 & HSP90AA1 & Heat shock protein HSP 90-alpha & Targetnet(0.757) \\
\hline 28 & NOS2 & Niric oxide synthase, inducible & Targetnet $(0.384)$ \\
\hline
\end{tabular}

\subsection{PPI network analysis}

The Cytoscape software was used to map the PPI network of 28 potential targets (Fig. 3A). PTPRS and ERAP1 were excluded as the results showed they were isolated. Further analysis of the remaining 26 targets revealed that the PPI contained 26 nodes and 83 edges (Fig. 3B). Among these nodes, the degree values of IL-6, TNF, VEGFA, HSP90AA1, CREB1, IL2, FGF2, ABCB1, NRAS, TYR, NOS2, and ACHE were greater than the median (5). They were suggested to play an important role in the anti-osteoporosis activity of vaccarin. Moreover, the degree values of IL-6, TNF, VEGFA, HSP90AA1, CREB1, and IL-2 were more than twice the median value; hence, they could be the key targets of vaccarin against osteoporosis (Table 2). 
Table 2

Protein-protein interaction at common targets of vaccarin and osteoporosis

\begin{tabular}{|c|c|c|c|}
\hline No. & Potential target & Degree & Closeness centrality \\
\hline 1 & IL6 & 18 & 0.75757576 \\
\hline 2 & TNF & 14 & 0.65789474 \\
\hline 3 & VEGFA & 14 & 0.65789474 \\
\hline 4 & HSP90AA1 & 12 & 0.625 \\
\hline 5 & CREB1 & 11 & 0.625 \\
\hline 6 & IL2 & 10 & 0.58139535 \\
\hline 7 & FGF2 & 9 & 0.56818182 \\
\hline 8 & ABCB1 & 9 & 0.54347826 \\
\hline 9 & NRAS & 8 & 0.52083333 \\
\hline 10 & TYR & 7 & 0.49019608 \\
\hline 11 & NOS2 & 6 & 0.5 \\
\hline 12 & $\mathrm{ACHE}$ & 6 & 0.54347826 \\
\hline 13 & FGF1 & 5 & 0.49019608 \\
\hline 14 & LGALS3 & 5 & 0.5 \\
\hline 15 & DNMT1 & 5 & 0.52083333 \\
\hline 16 & PTGS1 & 5 & 0.49019608 \\
\hline 17 & CYP1B1 & 4 & 0.48076923 \\
\hline 18 & $\mathrm{XDH}$ & 3 & 0.46296296 \\
\hline 19 & CYP1A2 & 3 & 0.37313433 \\
\hline 20 & ADRA2A & 3 & 0.41666667 \\
\hline 21 & $\mathrm{BCL} 2 \mathrm{~A} 1$ & 3 & 0.46296296 \\
\hline 22 & $\mathrm{P} 4 \mathrm{HB}$ & 2 & 0.45454545 \\
\hline 23 & NMUR2 & 1 & 0.29761905 \\
\hline 24 & CA2 & 1 & 0.34722222 \\
\hline 25 & ALPI & 1 & 0.35714286 \\
\hline 26 & FASN & 1 & 0.43859649 \\
\hline
\end{tabular}




\subsection{GO and KEGG pathway enrichment analyses}

GO annotation revealed that the expressed 28 potential targets were mainly associated with 23 different biological processes, including positive regulation of the apoptotic process, lung development, positive regulation of sprouting angiogenesis, epithelial cell proliferation involved in salivary gland morphogenesis, and nitric oxide biosynthetic process (Fig. 4). This result indicated that vaccarin could regulate multiple biological processes to prevent and treat osteoporosis.

The intersection targets were found to interact with 22 KEGG pathways mainly involving the PI3K/Akt signaling pathway (eight targets), pathways in cancer (seven targets), HTLV-1 infection (five targets), microRNA in cancer (five targets), Chagas disease (American trypanosomiasis) (four targets), hepatitis B (four targets), and tuberculosis (four targets) (Fig. 5A). These pathways were then cataloged into four groups: (1) affecting the differentiation and apoptosis of bone reconstruction-related cells (5 pathways), as represented by the PI3K/Akt and the Rap1 signaling pathways; (2) the estrogen signaling pathway correcting endocrine metabolic disorder (1 pathway); (3) inhibiting inflammatory response (2 pathways), as represented by the NOD-like receptor signaling pathway; and (4) 14 other disease signaling pathways, as represented by the cancer-signaling pathway (Fig. 5B). PI3K/Akt is the signaling pathway with the largest number of targets, including NRAS, IL6, HSP90AA1, CREB1, VEGFA, FGF1, FGF2, and IL2. Further analysis of the PI3K/Akt pathway in the KEGG database revealed that it might be closely related to osteoclast protein synthesis, proliferation, survival, cell cycle, and differentiation (Fig. 6). This analysis suggested that vaccarin may play a synergistic role in the prevention and treatment of osteoporosis by modulating multiple targets and pathways.

\section{Discussion}

The emerging field of network pharmacology can apply systematic research on the multitarget and multipathway characteristics of the compounds or monomers of Chinese herbal medicines and reveal them at the molecular level. Therefore, network pharmacology, a systems biology-based approach, provides great convenience and saves valuable time for researchers in conducting drug screening and validating disease-signaling pathways.

Previous studies reported that vaccarin has a wide range of biological effects, such as the induction of FGF2 expression and the promotion of angiogenesis [22]. In terms of clinical translational applications, wound dressings containing vaccarin can inhibit inflammatory cell infiltration [8] and effectively promote endothelial cell migration and wound healing [11]. However, the issue of whether vaccarin can prevent and treat osteoporosis by influencing these biological processes is not yet clear.

Six target proteins, namely, IL-6, TNF, VEGFA, HSP90AA1, CREB1, and IL-2, found in the PPI network analysis were considered to be the most critical targets for pharmacological intervention in osteoporosis. The occurrence of osteoporosis is a very complex pathological process that may involve several key 
target proteins. The monomers of Chinese herbal medicines, unlike small-molecule inhibition, often exert their therapeutic effects by affecting multiple targets. The biggest advantage of conducting a PPI network analysis is that the proteins with the greatest effect on gene interactions at the intersecting targets can be rapidly calculated, which in turn provides clear targets for drug intervention. Among the aforementioned key target proteins, IL-6, TNF, and IL-2 are important members of the inflammatory factor family, and they have been found to play critical roles in the differentiation of osteoclast precursor cells to mature osteoclasts[23]. VEGFA is a member of the vascular endothelial growth factor family, HSP90AA1 is an important member of the heat shock protein family [24], and CREB1 belongs to the important family of leucine zippers of DNA-binding proteins[25].

GO annotation analysis suggested that vaccarin might influence the pathogenesis of osteoporosis through 23 different biological processes, including regulation of apoptosis, angiogenesis, endothelial cell migration, lipolysis metabolism, insulin secretion, and cellular response to hydrogen peroxide. Several studies have proved that vaccarin plays a role in the treatment of other diseases by influencing the aforementioned biological processes. For instance, high glucose-induced endothelial cell injury can be reversed by downregulating the expression level of the apoptotic protein caspase 3[26]. Fibroblast growth factor receptor 1 activation can promote angiogenesis [19]. Disorders of glycolipid metabolism can be corrected by improving insulin resistance [11]. Increasing the activity of superoxide dismutase, catalase, and glutathione peroxidase in vivo can reduce the damage caused by $\mathrm{H}_{2} \mathrm{O}_{2}$ and oxidative stress to endothelial cells[9].

Notably, GO annotation analysis revealed positive regulation of osteoclast differentiation, which was inconsistent with the findings of previous studies that vaccarin negatively regulates osteoclast differentiation. This inconsistency may be attributed to the fact that network pharmacology can only predict the biological process in which the drug is involved, and the positive or negative regulation by the drug must be experimentally confirmed. Moreover, other biological processes in which vaccarin may be involved were identified, but extensive basic experiments are warranted to confirm them. Research on the potential signaling pathways of vaccarin in the treatment of osteoporosis mainly focused on the following four different pathological processes.

\section{Effects on osteogenic cell differentiation and apoptosis pathways}

Osteoblast apoptosis plays a crucial role in the pathogenesis of postmenopausal osteoporosis[27]. Both osteoblasts and osteoclasts are engaged in the process of bone reconstruction, and any influence on either side may break the balance inside the bone. PI3K/AKt is a signaling pathway closely related to cell proliferation and apoptosis, and PI3K/Akt signaling activation can reportedly promote osteoblast apoptosis [28]. Rap1 and Ras, which belong to the small GTPase family, are upstream signaling molecules of the MAPK signal and can be activated by various extracellular signals, such as growth factors, receptor tyrosine kinases, and $\mathrm{Ca} 2+$. Experimental studies have proved that Rap1 can promote osteoblast proliferation and differentiation by influencing the MAPK signaling pathway[29], whereas Ras activation can increase cyclinDI expression to promote osteoblast proliferation [30]. HIF-1a is also 
involved in osteoclast formation and can effectively prevent the occurrence of osteoporosis by inhibiting its expression [31].

\section{Internal secretion metabolic disorder}

Postmenopausal osteoporosis is a common endocrine metabolism disorder. As the ovaries stop producing estrogen and serum estrogen levels drop precipitously after menopause, the postmenopausal female experiences a state of high inflammation and oxidative stress. This situation leads to osteoclast formation promotion, osteoblast proliferation inhibition, and steady state of bone rebuilding disruption[15].

\section{Inflammatory response}

Numerous inflammatory factors and inflammation-related signaling pathways are involved in the pathogenesis of osteoporosis. NOD-like receptors have been found to activate cysteine proteases, upregulate the secretion of the inflammatory factor IL-1B, and exacerbate inflammation-induced pathological bone loss[32].

\section{Pathways associated with other diseases}

The present KEGG pathway enrichment analysis revealed that rheumatoid arthritis, prostate cancer, tuberculosis, and amoebiasis may interact with osteoporosis. Overactive osteoclasts in advanced rheumatoid arthritis cause bone resorption, resulting in severe destruction of cartilage and subchondral bone[33]. Bone metastasis, which is different from general malignant tumor metastasis, is most common in advanced prostate cancer. Bone tuberculosis often occurs in bones or joints that bear heavy weights and are thus prone to damage, with the greatest damage being joint deformity and nerve compression due to bone destruction. Both of these disease processes are closely related to osteoclast-induced bone resorption. Thus, signaling pathways associated with rheumatoid arthritis, prostate cancer, tuberculosis, and other diseases may also be targets for vaccarin in the treatment of osteoporosis.

\section{Conclusion}

Through network pharmacology, we predicted that IL-6, TNF, VEGFA, HSP90AA1, CREB1, and IL2 might be the key targets of vaccarin against osteoporosis. The potential mechanisms of action of vaccarin against osteoporosis were based on various biological functions, such as regulation of apoptosis, positive regulation of neovascularization, negative regulation of lipolysis metabolic process, and positive regulation of osteoclast differentiation. KEGG pathway enrichment analysis revealed that vaccarin mainly treats osteoporosis by affecting the differentiation and apoptosis of bone rebuilding cells, correcting endocrine metabolic disorders, inhibiting inflammatory responses, and exerting therapeutic effects through interactions with other diseases. On the basis of these results, we propose that the antiosteoporosis activity of vaccarin is mainly based on the direct or indirect modulation of the 
aforementioned potential targets and pathways. These results provide a new therapeutic approach for the treatment of postmenopausal osteoporosis.

\section{Abbreviations}

PPI: Protein-Protein Interaction; GO: Gene ontology; KEGG: Kyoto encyclopedia of genes and genomes

\section{Declarations}

\section{Authors' contributions}

$\mathrm{YL}, \mathrm{XJC}$ and SJL designed the research. WYF and CSL performed the research, $\mathrm{YL}$ and $\mathrm{XJC}$ analyzed the data, MYB, YD and YL wrote the manuscript. All authors participated in the preparation of the manuscript. All authors read and approved the final manuscript.

\section{Funding and acknowledgements}

This work was partly supported by the National Natural Science Foundation of China (NSFC, No 81960768), the Natural Science Foundation of Guangxi Province (Nos 2017GXNSFBA198098 and 2019JJA140408) and Clinical research Climbing program of the First Affiliated Hospital of Guangxi Medical University (No 201903038).

\section{Ethics approval and consent to participate}

Not applicable.

\section{Consent for publication}

Not applicable.

\section{Availability of data and materials}

Not applicable.

\section{Competing interests}

All authors declare that they have no competing interests.

\section{Author details}

${ }^{1}$ Center for Genomic and Personalized Medicine, Guangxi Medical University, Nanning 530021, Guangxi, China;

${ }^{2}$ Guangxi key Laboratory for Genomic and Personalized Medicine, Nanning 530021, Guangxi, China; 
${ }^{3}$ Guangxi Collaborative Innovation Center for Genomic and Personalized Medicine, Nanning 530021, Guangxi, China;

${ }^{4}$ Guangxi Key Laboratory of Colleges and Universities, Nanning 530021, Guangxi, China;

${ }^{5}$ First Clinical Medical College, Guangzhou University of Chinese Medicine, Guangzhou 510400, Guangdong, China;

${ }^{6}$ Departments of Orthopedics, The First Affiliated Hospital of Guangxi Medical University, Nanning 530021, Guangxi, China;

University, Nanning 530021, Guangxi, China;

${ }^{7}$ The Second Affiliated Hospital of Guangxi Medical University, Nanning 530000, Guangxi, China;

8 Institute of Urology and Nephrology, First Affiliated Hospital of Guangxi Medical University, Nanning 530021, Guangxi, China

\section{References}

1. LI-Fan, Liang JYJSPJ. Research progress of Vaccaria segetalis. 2007.

2. Li SJCIBTC. Compendium of Materia Medica (Bencao Gangmu). 2006.

3. Sang S, Lao A, Chen Z, Uzawa J, And YF. Chemistry and Bioactivity of the Seeds of Vaccaria segetalis. 2003.

4. Zhang H, Wang K, Wu J, Chen Y, He P. A new flavonoid glycoside from Vaccaria hispanica. Natural product communications. 2011;6(11):1599-602.

5. Yang G, Zhang N, Wang T, et al. Liquid chromatography-tandem mass spectrometric assay for the determination of vaccarin in rat plasma: application to a pharmacokinetic study. Biomedical chromatography : BMC. 2014;28(12):1789-94.

6. Xie F, Cai W, Liu Y, et al. Vaccarin attenuates the human EA.hy926 endothelial cell oxidative stress injury through inhibition of Notch signaling. International journal of molecular medicine. 2015;35(1):135-42.

7. Qiu Y, Qiu L, Cui J, Wei Q. Bacterial cellulose and bacterial cellulose-vaccarin membranes for wound healing. Materials science \& engineering C, Materials for biological applications. 2016;59:303-9.

8. Xie F, Feng L, Cai W, et al. Vaccarin promotes endothelial cell proliferation in association with neovascularization in vitro and in vivo. Molecular medicine reports. 2015;12(1):1131-6.

9. Gong L, Lei Y, Liu Y, et al. Vaccarin prevents ox-LDL-induced HUVEC EndMT, inflammation and apoptosis by suppressing ROS/p38 MAPK signaling. American journal of translational research. 2019;11(4):2140-54. 
10. Hou B, Cai W, Chen T, et al. Vaccarin hastens wound healing by promoting angiogenesis via activation of MAPK/ERK and PI3K/AKT signaling pathways in vivo. Acta cirurgica brasileira. 2020;34(12):e201901202.

11. Liu Y, Sun J, Ma X, et al. Vaccarin Regulates Diabetic Chronic Wound Healing through FOXP2/AGGF1 Pathways. International journal of molecular sciences. 2020;21(6).

12. Lei $Y$, Gong L, Tan F, et al. Vaccarin ameliorates insulin resistance and steatosis by activating the AMPK signaling pathway. European journal of pharmacology. 2019;851:13-24.

13. Cai W, Zhang Z, Huang Y, Sun H, Qiu L. Vaccarin alleviates hypertension and nephropathy in renovascular hypertensive rats. Experimental and therapeutic medicine. 2018;15(1):924-32.

14. Liu Y, Song FM, Ma ST, et al. Vaccarin prevents titanium particle-induced osteolysis and inhibits RANKL-induced osteoclastogenesis by blocking NF-KB and MAPK signaling pathways. Journal of cellular physiology. 2019;234(8):13832-42.

15. Sapir-Koren R, Livshits G. Postmenopausal osteoporosis in rheumatoid arthritis: The estrogen deficiency-immune mechanisms link. Bone. 2017;103:102-15.

16. Hopkins ALJNB. Network pharmacology. 2007;25(10):1110-1.

17. Gan D, Xu X, Chen D, Feng P, Xu Z. Network Pharmacology-Based Pharmacological Mechanism of the Chinese Medicine Rhizoma drynariae Against Osteoporosis. Medical science monitor : international medical journal of experimental and clinical research. 2019;25:5700-16.

18. Wang N, Xu P, Wang X, et al. Integrated pathological cell fishing and network pharmacology approach to investigate main active components of Er-Xian decotion for treating osteoporosis. Journal of ethnopharmacology. 2019;241:111977.

19. Liu WJ, Jiang ZM, Chen Y, et al. Network pharmacology approach to elucidate possible action mechanisms of Sinomenii Caulis for treating osteoporosis. Journal of ethnopharmacology. 2020;257:112871.

20. Zhou Y, Zhou B, Pache L, et al. Metascape provides a biologist-oriented resource for the analysis of systems-level datasets. Nature communications. 2019;10(1):1523.

21. Chen L, Zhang YH, Wang S, Zhang Y, Huang T, Cai YD. Prediction and analysis of essential genes using the enrichments of gene ontology and KEGG pathways. PloS one. 2017;12(9):e0184129.

22. Sun HJ, Cai WW, Gong LL, et al. FGF-2-mediated FGFR1 signaling in human microvascular endothelial cells is activated by vaccarin to promote angiogenesis. Biomedicine \& pharmacotherapy = Biomedecine \& pharmacotherapie. 2017;95:144-52.

23. Trzeciak-Ryczek A, Tokarz-Deptuła B, Deptuła W. Expression of IL-1Ra, IL-6, IL-8, IL-18, TNF-a and IFNY genes in peripheral blood leukocytes of rabbits infected with RHDV (Rabbit Haemorrhagic Disease Virus). Developmental and comparative immunology. 2017;76:310-5.

24. Yang $C$, Huang D, Ma C, et al. Identification of Pathogenic Genes and Transcription Factors in Osteosarcoma. Pathology oncology research : POR. 2020;26(2):1041-8. 
25. Yu C, Bai YX, Xu XP, et al. Behavioral Abnormality along with NMDAR-related CREB Suppression in Rat Hippocampus after Shortwave Exposure. Biomedical and environmental sciences : BES. 2019;32(3):189-98.

26. Qiu Y, Du B, Xie F, et al. Vaccarin attenuates high glucose-induced human EA•hy 926 endothelial cell injury through inhibition of Notch signaling. Molecular medicine reports. 2016;13(3):2143-50.

27. Guo TM, Xing YL, Zhu HY, Yang L, Liu GX, Qiao XM. Extracellular regulated kinase 5 mediates osteoporosis through modulating viability and apoptosis of osteoblasts in ovariectomized rats. Bioscience reports. 2019;39(9).

28. Zhang Y, Cao X, Li P, et al. PSMC6 promotes osteoblast apoptosis through inhibiting PI3K/AKT signaling pathway activation in ovariectomy-induced osteoporosis mouse model. Journal of cellular physiology. 2020;235(7-8):5511-24.

29. Pan BL, Tong ZW, Li SD, et al. Decreased microRNA-182-5p helps alendronate promote osteoblast proliferation and differentiation in osteoporosis via the Rap1/MAPK pathway. Bioscience reports. 2018;38(6).

30. Han XG, Wang DW, Bi ZG, Gao F. Regulatory effect of estrogen receptor-a-mediated Wnt/ $\beta$-catenin signaling pathway on osteoblast proliferation. Journal of biological regulators and homeostatic agents. 2016;30(2):381-7.

31. Zhong H, Cao C, Yang J, Huang Q. [Research on Relationship of HIF-1 Signaling Pathway and Postmenstrual Osteoporosis]. Sichuan da xue xue bao Yi xue ban = Journal of Sichuan University Medical science edition. 2017;48(6):862-8.

32. Hoseini Z, Sepahvand F, Rashidi B, Sahebkar A, Masoudifar A, Mirzaei H. NLRP3 inflammasome: Its regulation and involvement in atherosclerosis. Journal of cellular physiology. 2018;233(3):2116-32.

33. Adami G, Fassio A, Rossini M, et al. Osteoporosis in Rheumatic Diseases. International journal of molecular sciences. 2019;20(23).

\section{Figures}



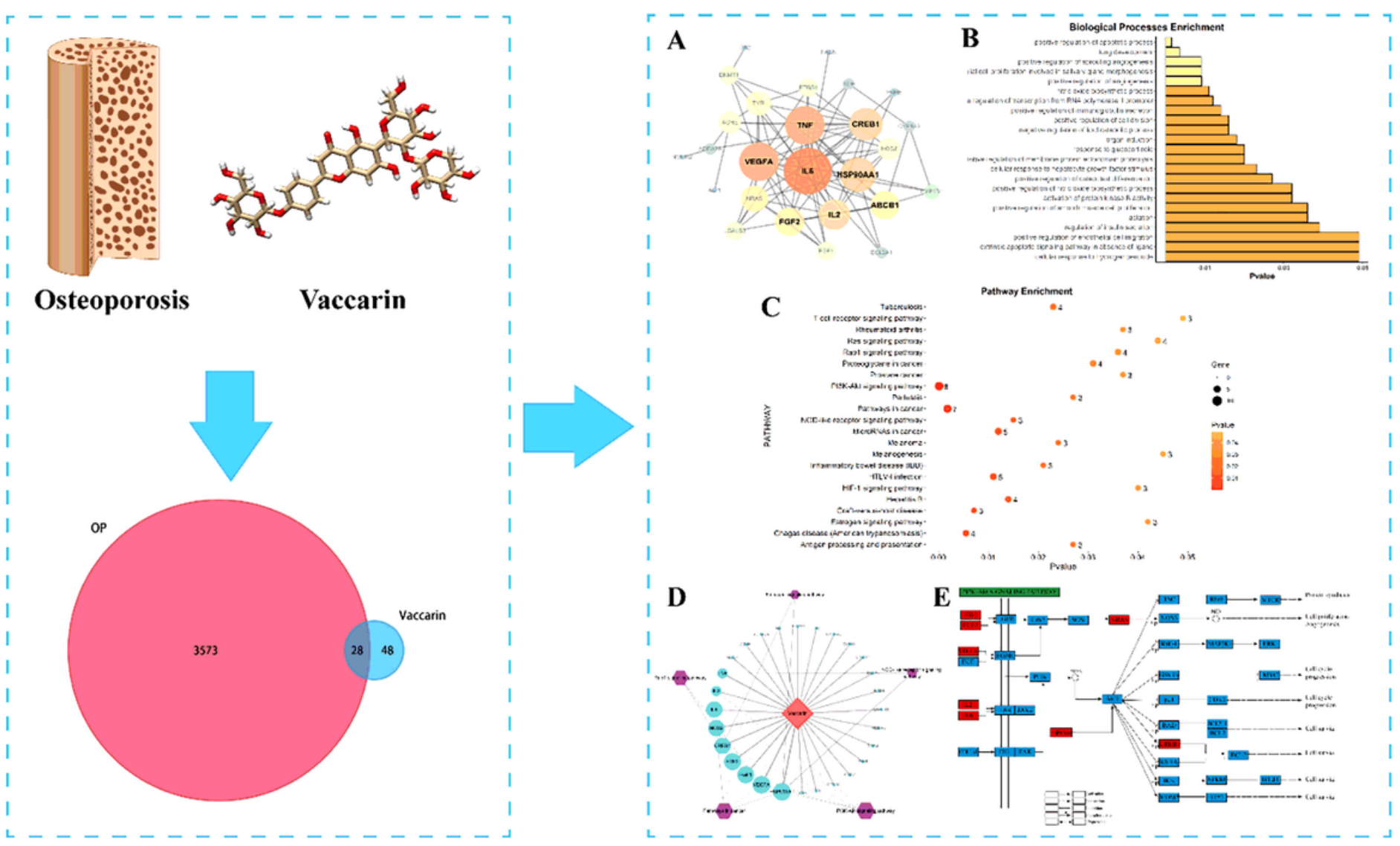

Figure 1

Flow chart of network pharmacology study on intervention of vaccarin in osteoporosis 


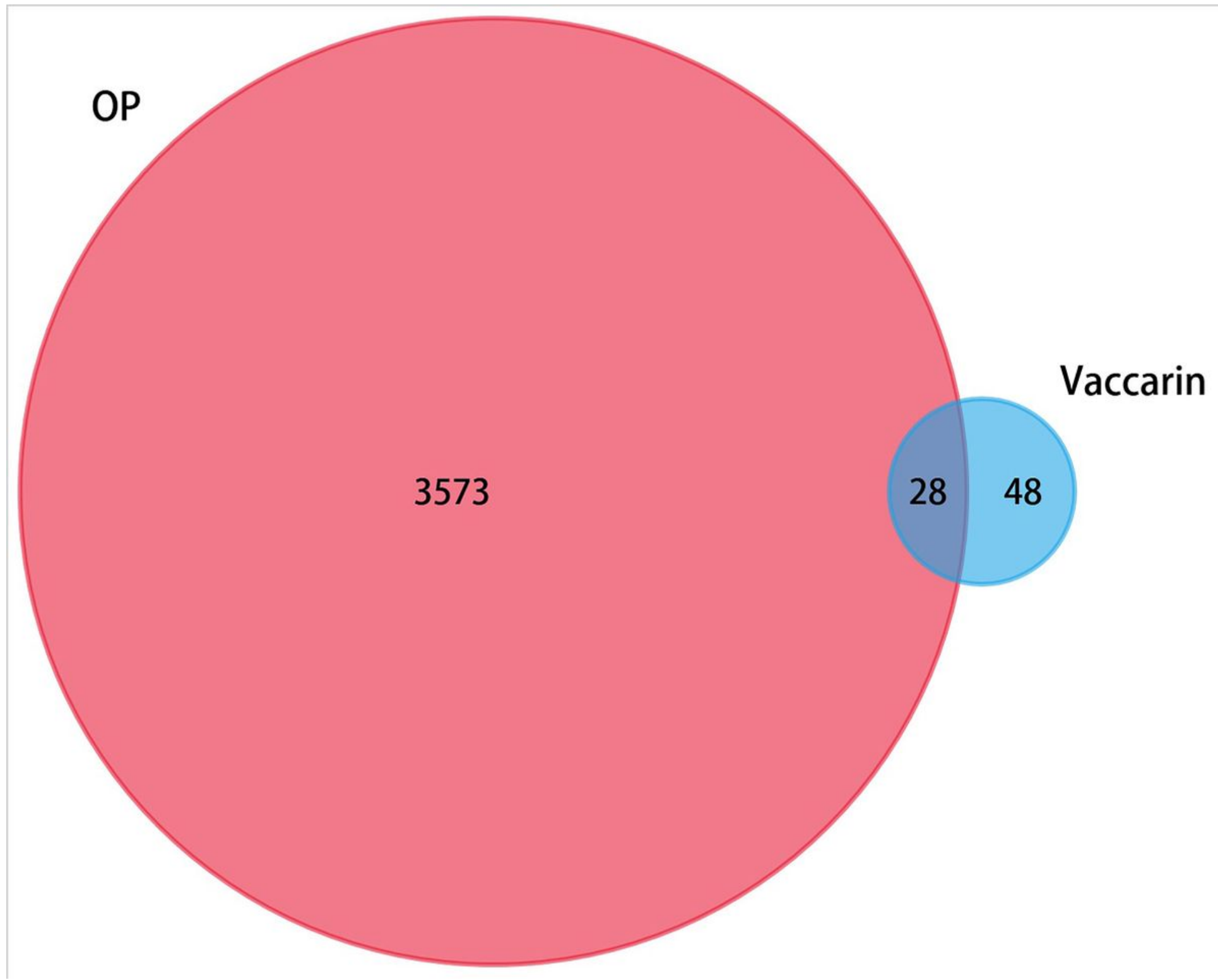

Figure 2

Venn diagram of the target genes of vaccarin and osteoporosis. 


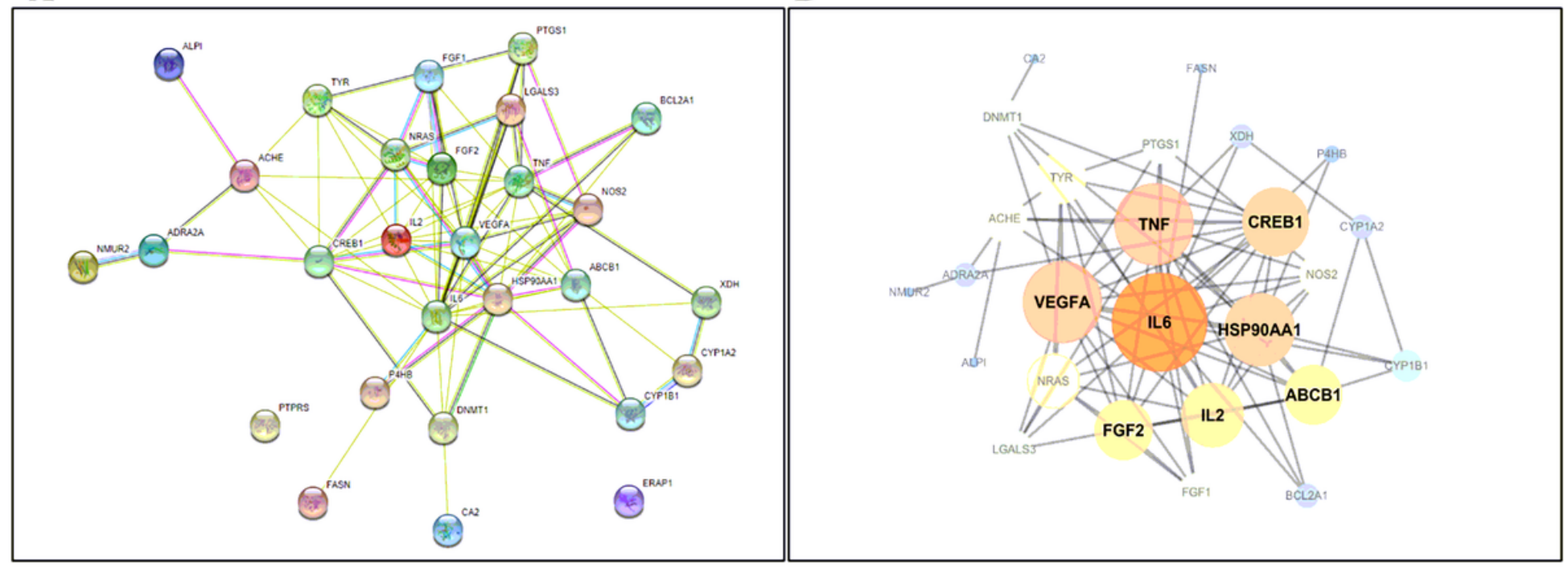

\section{Figure 3}

The protein interaction network of the combined action targets of vaccarin and osteoporosis. (A) PPI network of 28 targets. (B) PPI network after exclusion of isolated targets PTPRS, ERAP1.

\section{Biological Processes Enrichment}

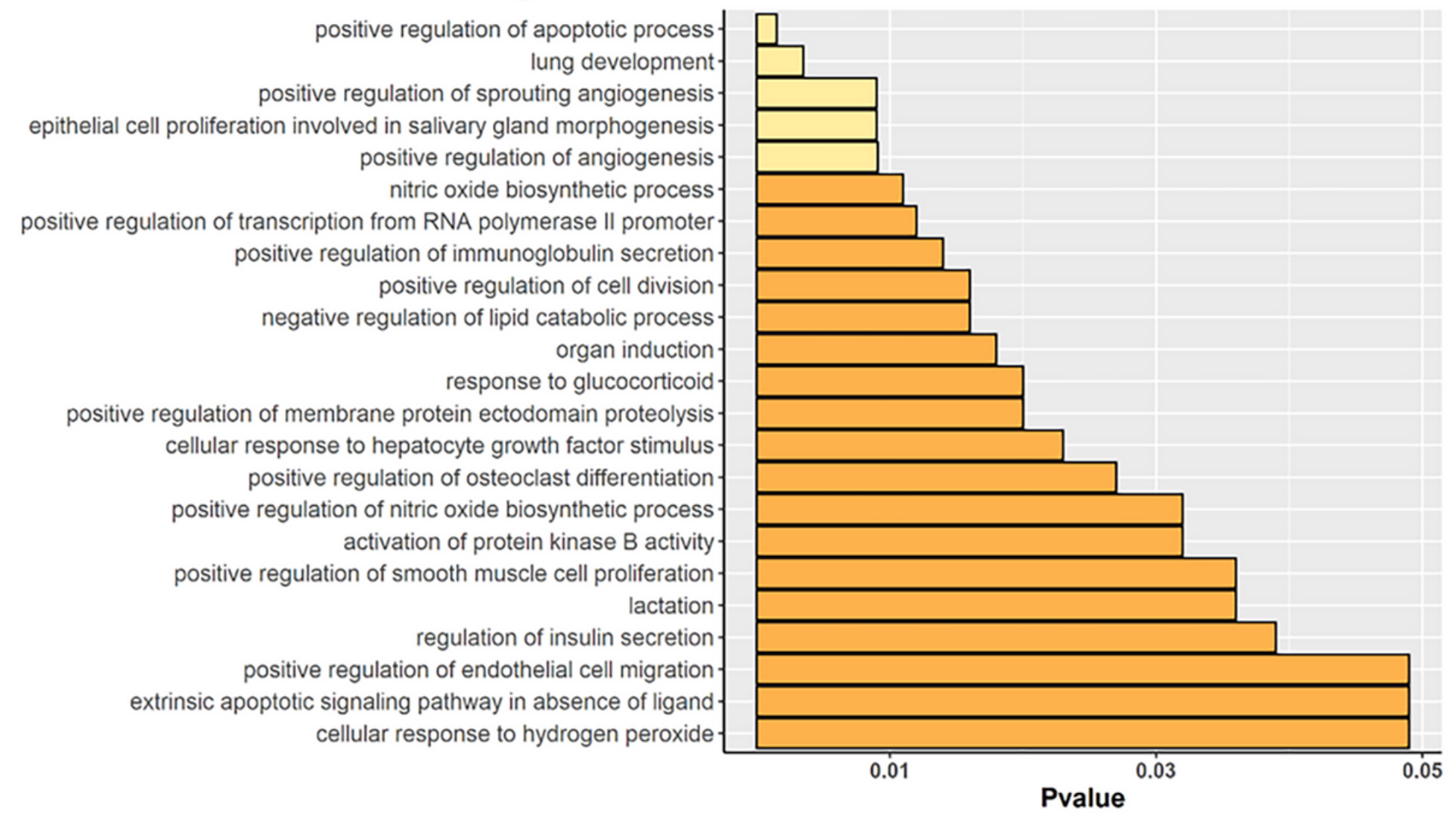

\section{Figure 4}

Biological process results of gene ontology annotations of intersection target proteins. 
A

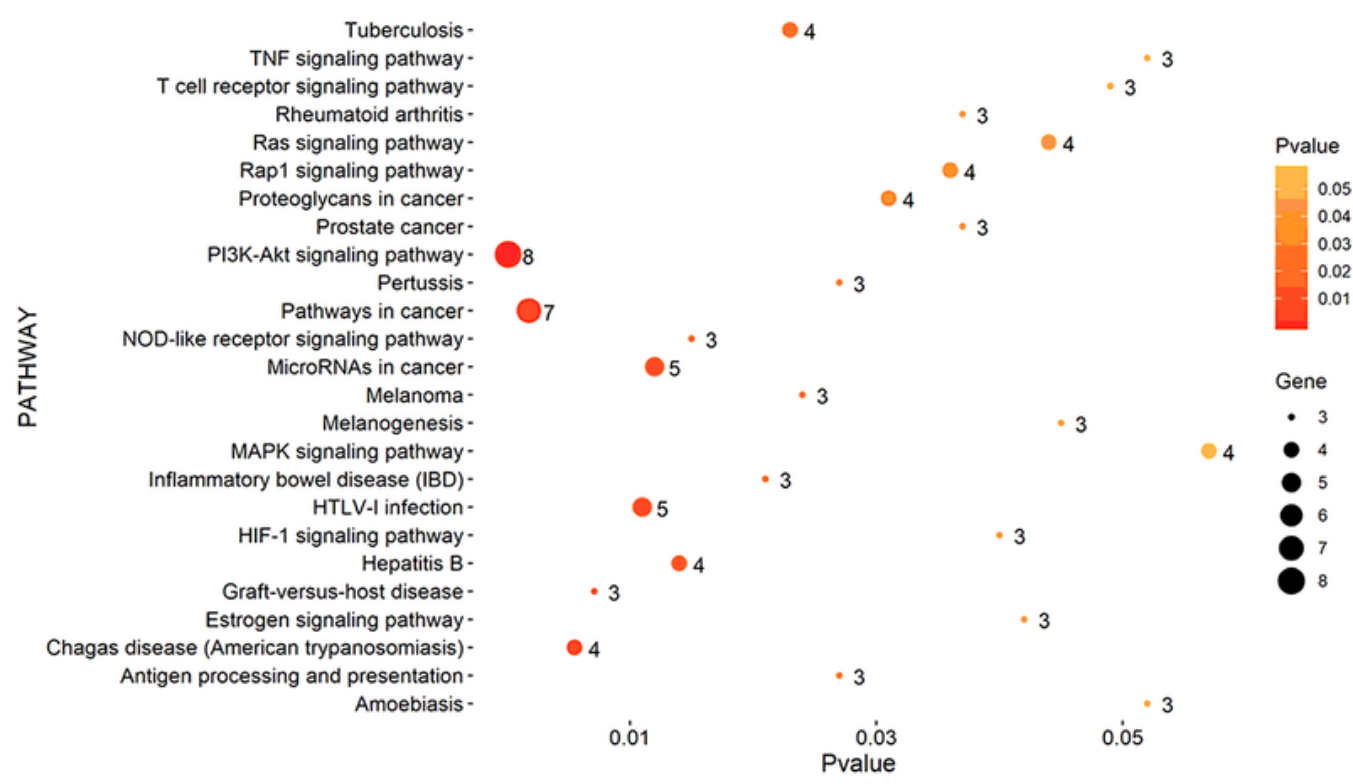

B

TNF signaling pathway

HIF-1 signaling pathway

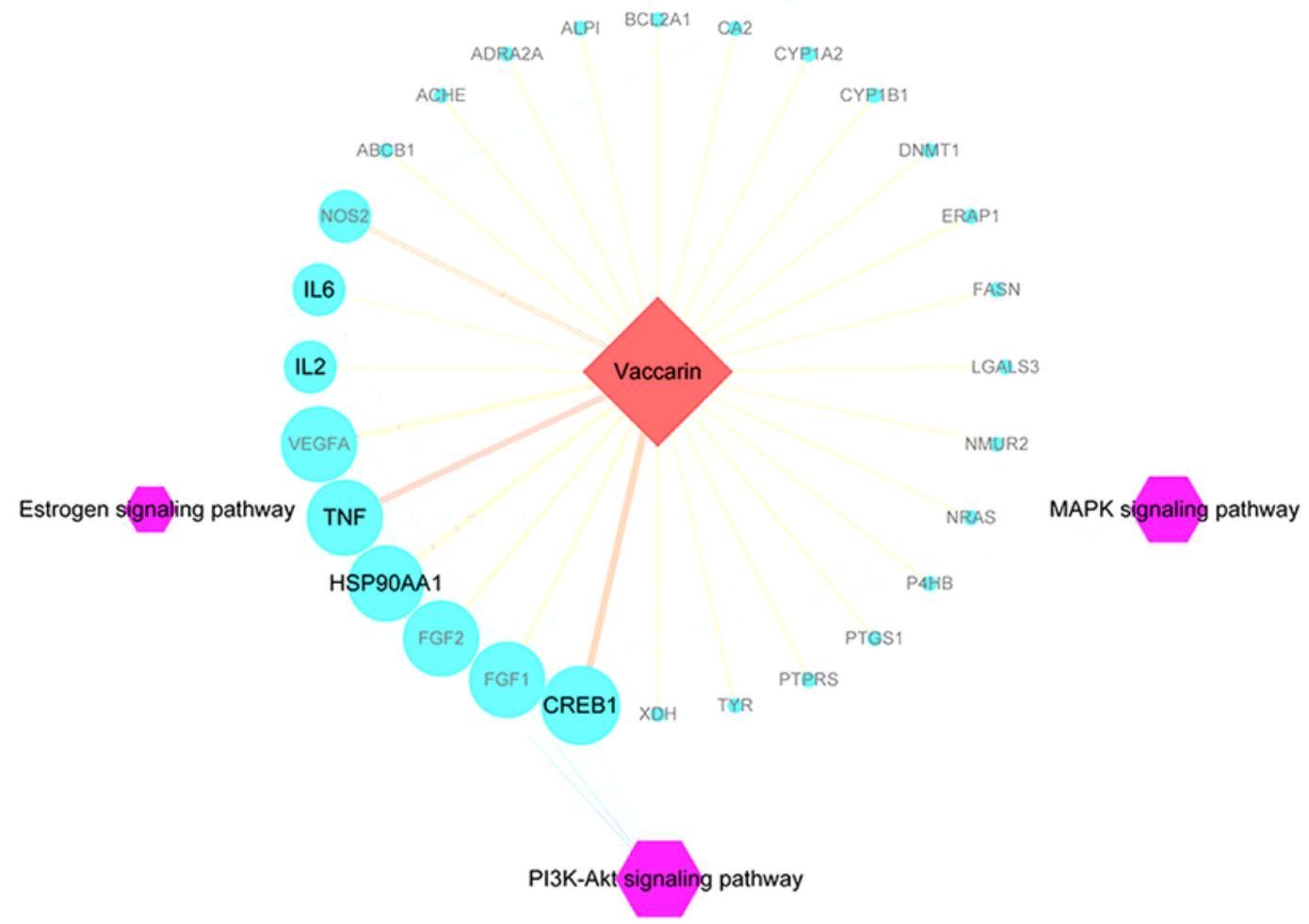

Figure 5

KEGG pathway enrichment analysis of the intersection targets of vaccarin and osteoporosis. (A) KEGG pathway enrichment analysis bubble map of intersecting targets. (B) Mapping of intersecting targets in the PI3K/Akt, cancer pathway, estrogen pathway, Rap1 pathway, and NOD-like receptor pathway. 


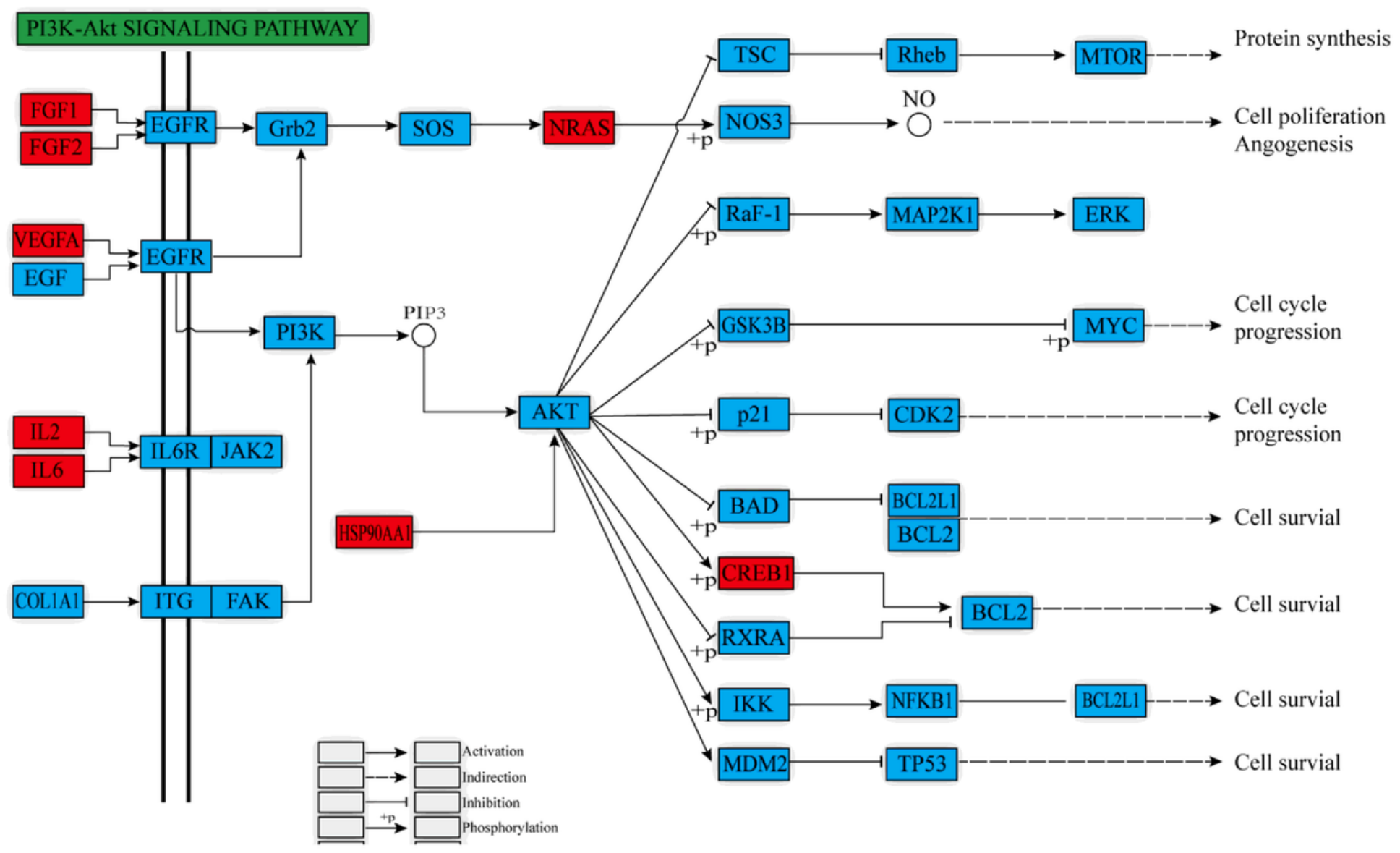

Figure 6

$\mathrm{PI} 3 \mathrm{~K} /$ Akt target-pathway network. The red nodes represent the hub genes, the green nodes represent other genes and the blue nodes are the genes of this pathway 